\title{
Autonomous Robotic SLAM-based Indoor Navigation for High Resolution Sampling with Complete Coverage
}

\author{
Iris Wieser*, Alberto Viseras Ruiz, Martin Frassl, \\ Michael Angermann, Joachim Mueller, Michael Lichtenstern \\ Institute of Communications and Navigation \\ German Aerospace Center (DLR) \\ Wessling, Germany \\ Email: *wieseri@online.de
}

\begin{abstract}
Recent work has shown the feasibility of pedestrian and robotic indoor localization based only on maps of the magnetic field. To obtain a complete representation of the magnetic field without initial knowledge of the environment or any existing infrastructure, we consider an autonomous robotic platform to reduce limitations of economic or operational feasibility. Therefore, we present a novel robotic system that autonomously samples any measurable physical processes at high spatial resolution in buildings without any prior knowledge of the buildings' structure. In particular we focus on adaptable robotic shapes, kinematics and sensor placements to both achieve complete coverage in hardly accessible areas and not be limited to round shaped robots. We propose a grid based representation of the robot's configuration space and graph search algorithms, such as Best-First-Search and an adaption of Dijkstra's algorithm, to guarantee complete path coverage. In combination with an optical simultaneous localization and mapping (SLAM) algorithm, we present experimental results by sampling the magnetic field in an a priori unknown office with a robotic platform autonomously and completely.
\end{abstract}

Index Terms-Autonomous Navigation, Holonomic Robotic Platform, Complete Coverage, Path Planning, Optical SLAM, Graph Search, Robot Sensing Systems, Magnetic Field.

\section{INTRODUCTION}

\section{A. Motivation}

The characteristics of physical phenomena in space are a valuable input source of information for many applications: They range from the domain of network coverage planning, which can be improved given a map of radio signal strength, to evacuation planning after an incident including chemical, biological, radioactive or nuclear material (CBRN), where extension and motion of the hazardous cloud need to be predicted. To obtain such information at high spatial resolutions without any initial knowledge of the environment, we aim to capture samples of an underlying physical process using autonomous mobile robots.

We are particularly interested in magnetic fields within building structures. It has been shown that the highly nonuniform perturbations of the Earth's magnetic field inside

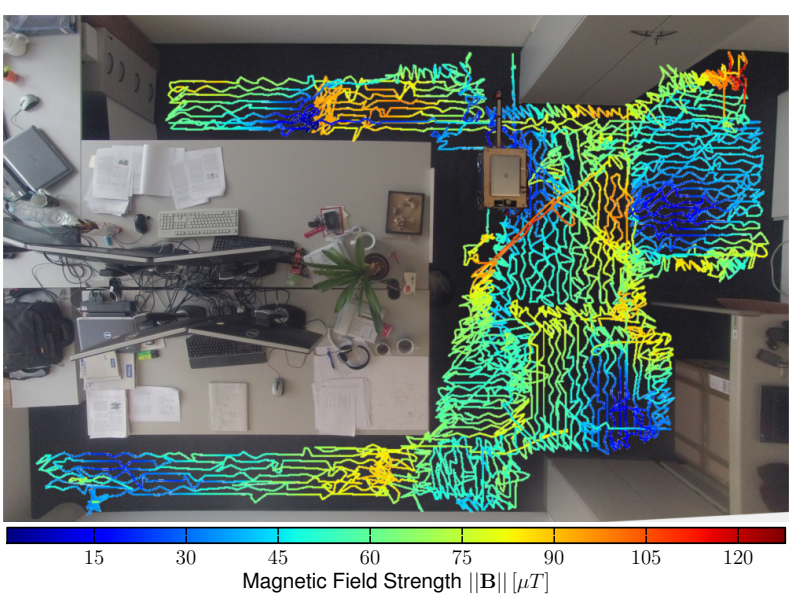

Fig. 1. Autonomous holonomic ground robot capturing the magnetic field with a spatial resolution of $0.05 \mathrm{~m}$ in an office at DLR with an overlayed trajectory estimation for the robotic platform by an optical SLAM algorithm. The color scale corresponds to the magnetic field intensity of the captured map.

buildings can be used for localization purposes. As the microstructured disturbances appear to be persistent and unique in terms of direction and intensity, the magnetic field has proven to be very rich in recognizable features [1]. This allows localization of both pedestrians and robots [2] with sensors that are affordable, portable and do not require any existing infrastructure. The foundation for the development and evaluation of such algorithms is ground truth information of the three-dimensional magnetic field map, which may be obtained by the approach described in this paper with autonomous mobile robots.

The limited perception ability of robots as well as the diversity of common indoor environments require a more flexible and adaptive path planning algorithm, as newly discovered obstacles in various geometric shapes need to be considered. Moreover, we are especially interested in capturing samples in hardly accessible areas (e.g. narrow spaces or corners). Therefore, we formulate and propose a system that is 


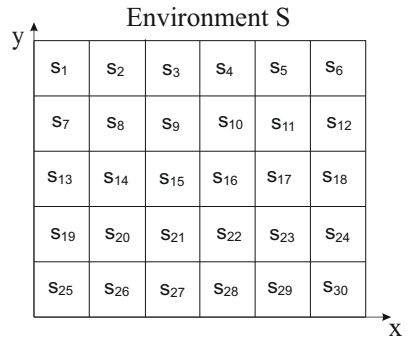

(a) Grid-based representation of the environment $S$.

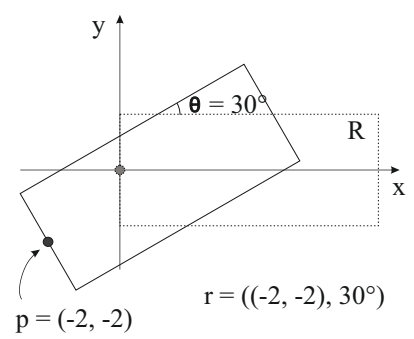

(b) Robot's pose $r=(p, \theta)=$ $\left((-2,-2), 30^{\circ}\right)$.
Fig. 2. Illustration of the environment and the robot's position and orientation.

independent of the robot's geometry as well as motion model. Furthermore, we aim to achieve (i) a complete representation of the physical process, (ii) collision avoidance with obstacles in the explored environment and (iii) efficiency in terms of exploration time.

\section{B. Problem Statement}

We want to obtain a complete representation of physical phenomena (such as the magnetic field) in an a priori unknown environment by an autonomous robotic platform in a minimum amount of time, while avoiding any collisions.

We formulate the model and algorithm for a twodimensional space in order to avoid notational clutter. The scenario is composed of the environment, in which the platform operates, the physical process, that is to be observed, and the robotic platform itself.

The environment is divided into a regular grid of $N_{s}$ cells, and therefore, represented by the set of cells $S=$ $\left\{s_{i}\right\}_{i \in\left[1, \ldots, N_{s}\right]}$ (see Fig. 2a). Each cell $s_{i, i \in\left\{1, \ldots, N_{s}\right\}}$ has a state, which is either free or occupied. If no obstacle is located in $s_{i}$, we define the cell as free. The set of cells that are considered as free, is called free space $S_{\text {free }}$. The occupied space $S_{o c c}$ is composed of the set of the remaining cells.

We define the physical process as $M=\left\{m_{i}\right\}_{i \in\left[1, \ldots, N_{s}\right]}$, with the physical quantity $m_{i}$ located in $s_{i}$. We assume the spatial frequency of the process to be low enough compared to the cell size, so that a single measurement inside a cell provides a sufficient estimation of the process at the cell's location.

The robotic platform is completely defined by the robot's pose $r=(p, \theta)$, as shown in Fig. $2 \mathrm{~b}$, with $p$ being the robot's spatial position with $p \in \mathbb{R}^{2}$ and $\theta$ representing the robot's independent rotational transformation with $\theta \in\left[0^{\circ}, 360^{\circ}\right.$.

We desire to develop a system that allows the robotic platform to take at least one sample $m_{i}$ within each free cell $s_{i}$ in a minimum amount of time without entering occupied space.

\section{Related Work}

Coverage path planning is a very essential component in numerous autonomous mobile robot applications, such as floor cleaning, lawn mowing, area inspection, mine sweeping and surveillance. Some of the first approaches for coverage path planning [3] included heuristic and randomized components. Encouraged by their simplicity and robustness, heuristic approaches can save costly localization sensors as well as computational resources. However, random walks cause path overlap and require more time to complete the task.

Another disadvantage of heuristic approaches relies in the not guaranteed full coverage. To achieve guaranteed coverage, Huang [4] introduced a divison of the coverage region in subregions and studies the optimal sweep direction for covering each subregion. Moreover, Yang and Luo [5] have shown a neural network approach for complete coverage path planning, that is especially distinguished by its computational simplicity. They describe the environment as the dynamic activity landscape of the neural network. However, the model requires complete knowledge of the environment in advance and assumes noisefree sensors.

Most applications, such as cleaning robots [6], usually imply large footprints with a given symmetrically shaped robot to cover an area. Viet et al. [7] utilize a commercial round shaped vacuum cleaning robot by iRobot to experimentally demonstrate a coverage path planning algorithm, in which critical points are used as backtracking points for a new bousdrophedon motion.

In contrast, we propose a system that is easily adaptable to any geometric shape and kinematic of a robot as well as its sensor's placement. As the footprint size of our utilized sensor is assumed to be infinitesimally small, an interpolation of measured points might be applied to achieve a complete representation.

K. Thiayagarajan and C. G. Balaji [8] achieve complete coverage with a minimum path overlap by a graph traversal algorithm. However, they assume full knowledge of the robot's environment and compute the path offline.

Our system does not require an a priori map of the environment, as a map of the environment is generated online during the experiment by simultaneous localization and mapping (SLAM). Also, the path planning algorithms are calculated at real time. To perceive the robot's workspace, it has been proven to be very successful to combine wheel encoder and optical sensors, such as laser scanner or cameras, in a SLAM algorithm. Grisetti, Stachniss and Burgard [9] have contributed an algorithm based on Rao-Blackwellized Particle Filter to enable an optical SLAM algorithm with decreased numbers of particles required to build a map substantially. Based on this approach, we propose a system to capture samples of physical quantities at high spatial resolution by a sensor with an infinitesimally small footprint within unstructured environments, of which we do not have a priori knowledge. The proposed system achieves complete coverage and is adaptable for any robotic geometry and kinematics.

The remainder of this paper is organized as follows. In Section II the overall system is presented with its components, such as coverage path planning, an efficient state space representation and an optical SLAM algorithm. Section III describes our experimental setup for enabling a mobile robotic platform to explore an environment autonomously using a laser scanner. 


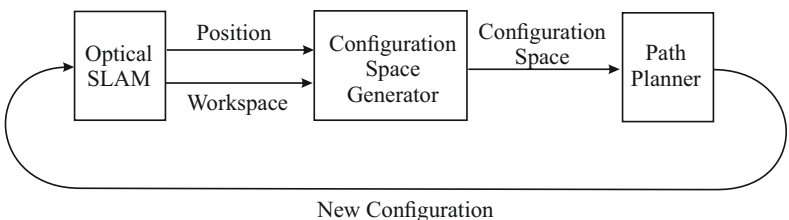

Fig. 3. Sequence diagram of proposed system. The optical SLAM algorithm estimates the current position and orientation of the robot and generates a map of the observed environment (workspace). A configuration space is created to allow an efficient path planning algorithm that calculates the next best configuration for the robot to navigate to.

Experiments carried out by capturing the magnetic field within an office at DLR are discussed in Section IV to support the validity of our system. Finally, in Section V conclusions and directions for further work are drawn.

\section{Models AND Algorithms}

Working with autonomous mobile robots involves managing and synchronizing four main tasks: perceiving the environment, localizing the robot within that environment, planning the next motion and executing the planned task. Fig. 3 illustrates the main sequence of the proposed system, which will be explained in the following sections in more detail.

Precise localization is required for acquiring a map of the environment (workspace) as well as an adequate robot control. In this paper, rotary encoders are utilized for the robot's odometry. However, an estimation error is accumulated over time, which results in a significant drift. Therefore, we utilize a SLAM algorithm to improve the localization by detecting recognizable features. The SLAM algorithm [9] is based on a Rao-Blackwellized Particle Filter, that enables grid map learning for laser scan data.

To guarantee complete coverage, we present a fine-grid based representation of the environment $S$ (see Fig 2a). Approximating the environment by cells with the same size and shape, each free cell needs to be passed at least once for complete coverage.

\section{A. Robot Model}

The robotic platform is modeled as an n-sided polygon $R$, defined by the tuple $P:=\left(P_{1}, P_{2}, . ., P_{n}\right), P_{h} \in \mathbb{R}^{2}, 1 \leq h \leq$ $n$. In Fig. 4 a the polygonal base is shown, that describes the robotic platform utilized in our experiments (see Fig. 4b). Due to a grid based representation of the environment, the robot's configuration $q_{i, k}$ can be defined by $q_{i, k}=\left(s_{i}, \theta_{k}\right)$, with $s_{i}$ being the corresponding cell to the robot's current position $p$.

For path planning we consider $p$ as the sensor's spatial position to capture the physical process under study. $\theta_{k}$ represents the robot's independent rotational transformation. Let $k, N_{\theta}$ define a quantization of one complete rotation, so that $\theta_{k}=k \cdot \frac{2 \pi}{N_{\theta}}$ with $k=1, \ldots, N_{\theta}$. The robot's body $B\left(q_{i, k}\right)$ is considered as the set of cells of the environment occupied by the robot with the configuration $q_{i, k}$, so that $B\left(q_{i, k}\right)=\left\{s_{i}\right\}_{s_{i} \in R, s_{i} \in S}$.

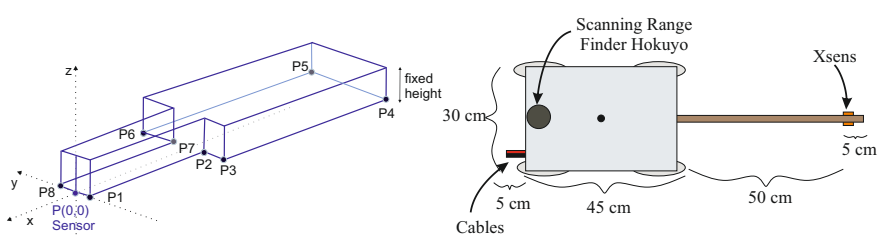

(a) Polygonal base of robot.

(b) Geometric description of robotic platform and its sensors.

Fig. 4. Models of robotic platform utilized in our experiments.

\section{B. Optical SLAM}

Our robot is equipped with an optical sensor which enables to perceive parts of the environment, depending on the sensor's position and orientation. A SLAM algorithm estimates the robot's current configuration by matching optical features to its previously obtained map. Let the set of cells that have been already perceived be stored in the robot's workspace $W$ with $W \subset S$. We define $\left\{\hat{S}_{i}\right\}$ as the set of cells newly observed by the optical sensor, with $\left\{\hat{S}_{i}\right\} \subset S$. Given those observations, the updated workspace is $\check{W}=W \cup\left\{\hat{S}_{i}\right\} . W$ is initialized as an empty set.

An additional sensor is mounted on the robotic platform to measure the physical process under investigation. The robot updates its set of measurements $Z$ by the new measurement $\left\{m_{a}, s_{a}\right\}$, with $m_{a}$ defined as the most recent measurement of the physical process taken within the cell $s_{a}$ corresponding to the estimated spatial position of the sensor. The updated set of measurements is $\check{Z}=Z \cup\left\{m_{a}, s_{a}\right\}$. The set of measurements $Z$ is initialized as an empty set.

\section{Configuration Space Generator}

An efficient representation of the robot's workspace is essential to synchronize between perception and planning. This representation needs to be compact, eligible to plan in, memory-efficient and - most important - complete, i.e. hold all desired information.

Based on the workspace $W$ a configuration space $C$ is calculated, that combines the kinematic and geometric aspects of the problem in one single description. Considering $C$ as a space that holds all possible configurations of the robot, it provides an abstraction of all reachable spatial cells $s_{i}$. We define the configuration space as $C=\left\{q_{i, k}\right\}=$ $\left\{s_{i}, \theta_{k}\right\}_{k=1, \ldots, N_{\theta}, s_{i} \in W}$.

Each configuration $q_{i, k}$ is specified in the configuration space as safe or unsafe, and if it is safe, as sensed or pending. This enables an efficient path planning, as only safe configurations are considered for path planning with the aim to reach all pending configurations. The set of safe configurations $C_{s}$ is defined as $C_{s}=\left\{q_{i, k} \mid B\left(q_{i, k}\right) \cap S_{o c c}=\emptyset\right\}$. If any intersection exists between an obstacle and the robot's body $B\left(q_{i, k}\right)$, the configuration $q_{i, k}$ is considered as unsafe. The set of pending configurations $C_{p}$ holds all configurations associated to a cell, that is safe and has not been measured yet, with $C_{p}=\left\{q_{i, k}\right\}_{q_{i, k} \in C_{S},\left(m_{i}, s_{i}\right) \notin Z}$. 


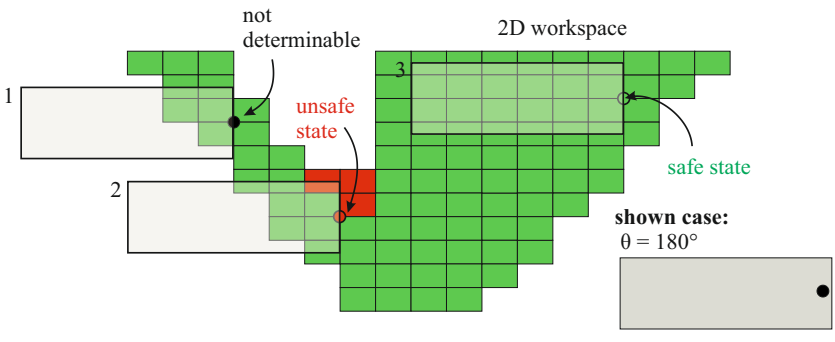

Fig. 5. Illustration of the currently perceived workspace (green rectangles show free space, red illustrate occupied space). The remaining environment is still unknown. It shows the determination of states, illustrating only states with $\theta=180^{\circ}$ for a rectangular robot model.

Fig. 5 illustrates a safe state, an unsafe state and an indeterminable state, as the workspace occupied by the robot's body $B\left(q_{i, k}\right)$ is still partly unknown.

The computation time needed to determine the set of cells occupied by $B\left(q_{i, k}\right)$ is strongly increased not only by the robot's rotational offset to the workspace's grid, but also the shape of the robot's polygon. However, we assume a static polygonal description of the robotic platform with discretized orientations $\theta_{k}$ and a grid-based representation of the environment. Therefore, all set of cells occupied by the robot are calculated beforehand for each possible orientation $\theta_{k}$ independent of the robot's translational transformation and are stored for later determinations of the configurations. As the workspace changes during the experiments, all states of the configurations need to be constantly updated, e.g. if human enters the sensor's view.

The configuration space is represented as a weighted graph with each configuration considered as a vertex. Configurations are connected by edges, if they are safe and no intermediate configuration is visited by the robot's movement from one to the other. The edges' weights are derived by means of measuring the duration of rotational and translational movements. Therefore, efficiency in terms of exploration time can be optimized by minimizing the costs for accessing all nodes within the graph. Fig. 6 illustrates a graph for a configuration space in a grid-based representation of the environment. For this particular case, each vertex can have a maximum amount of 10 edges.

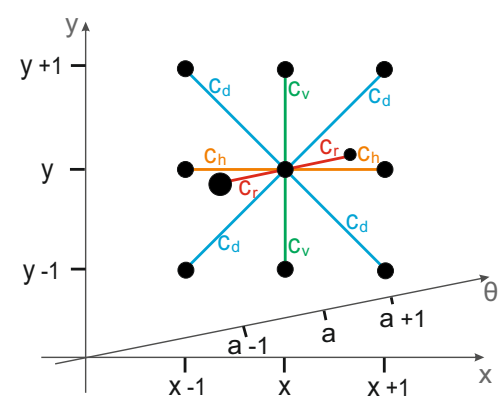

Fig. 6. Illustration of a vertex, its successors and their edges' costs $c_{d}, c_{h}, c_{v}, c_{r}$ for each possible transformation in the configuration space.
This setups allows the replacement of the ground-based robotic platform by simply exchanging the robot's geometric (polygonal model) and kinematic (costs) description.

\section{Path Planner}

Given the graph which represents the configuration space, we aim to calculate the robot's new configuration. Following a Best-First-Search approach we derive a low-complexity path planning algorithm to avoid time consuming calculations.

Let the robot's configuration $q_{i, k}$ correspond to the vertex $v\left(q_{i, k}\right)$. We define all pending vertices connected by an edge to the vertex $v\left(q_{i, k}\right)$ as its pending neighborhood. The vertex of this neighborhood offering the lowest cost, that is given by the edges' weights, is chosen as the robot's new configuration.

In case there are no pending vertices connected to the vertex $v\left(q_{i, k}\right)$, we apply an adaption of the Dijkstra-algorithm [10] to navigate the robot to the closest pending configuration, in terms of minimum costs. Incrementally summing up the costs from the inital vertex to each vertex, it will expand to its neighbors. However, the vertices with least costs are always expanded first until the first pending configuration is reached. To guarantee finding the vertex with the minimum total costs all vertices need to be further explored. The algorithm terminates if the summed up costs of each vertex is greater or equal to the cost of the first found pending configuration. The pending configuration explored with the least total costs to the inital vertex is chosen and its path will be applied to the robot.

The set of states $\left\{q_{i, k}\right\}_{k \in\left[1, \ldots, N_{\theta}\right]}$ is defined as sensed, as soon as a sample $m_{i}$ was taken at a position $p$ within the cell $s_{i}$. The algorithm is only terminated when no pending and reachable configurations exist anymore, so that the sensor has been placed in all perceived and free cells.

\section{EXPERIMENTAL SETUP}

For our experiments, we used a holonomic robotic platform and equipped it with a magnetic sensor to sense the physical process, as well as an optical range sensor to provide navigational information, see Fig. 7. We used a commercial laptop (with $8 \mathrm{~GB}$ of RAM at $1600 \mathrm{MHz}$ and a $2.1 \mathrm{GHz}$ Intel Core i7-4600U processor), which was carried on-board to analyze the data and run the algorithms.

We conducted experiments in two different locations, first in a lab equipped with an accurate ground truth system (optical Vicon tracking system) which we only utilized for the development and verification of our algorithms. In addition we carried out experiments in an office without external localization system. The results presented in this paper focus on the infrastructureless experiments.

\section{A. Robotic Platform}

The robotic platform employed is a customized version of the Slider150 (see Fig. 7), developed and manufactured by Commonplace Robotics $\mathrm{GmbH}$. Its outer dimensions are $0.45 \mathrm{~m} \times 0.3 \mathrm{~m} \times 0.17 \mathrm{~m}$. Due to its four mecanum wheels the platform is able to perform omnidirectional movements, 


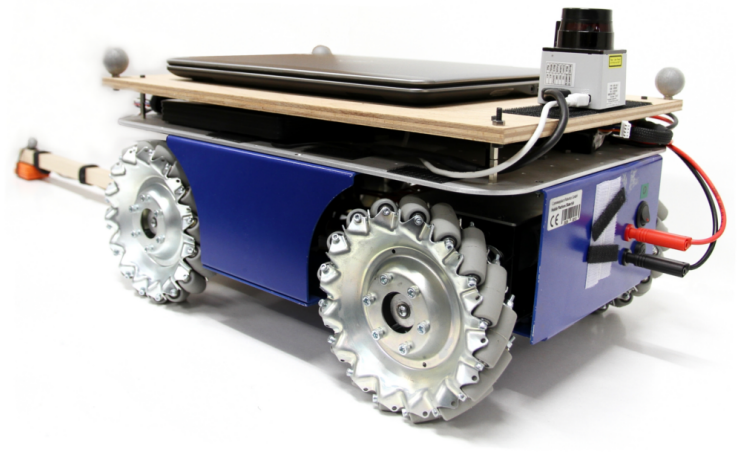

Fig. 7. Holonomic mobile robotic platform equipped with a laser scanner on top and a magnetic sensor extended on a wooden arm.

following input commands for forward, lateral and rotational velocities. Each of the four gear motors is equipped with a magnetic encoder that provides information about the rotational state of the motors at a rate of $10 \mathrm{~Hz}$, used to estimate the traveled distance.

\section{B. Magnetic Sensor}

To measure the magnetic field vector, we equipped the mobile platform with a wireless sensor unit, the XSens MTw. Besides the tri-axial magneto-resistive magnetometers, this commercially available sensor package incorporates accelerometers, gyroscopes and a barometer. We captured the sensor data with a rate of $100 \mathrm{~Hz}$. The sensor unit was installed on a wooden arm with a length of $0.75 \mathrm{~m}$. This construction minimizes undesired perturbations by the platform's ferromagnetic structures and electric currents. Additionally, it also enables measurements in otherwise hardly accessible locations of indoor environments, e.g. narrow spaces or corners. The sensor's placement is crucial for path planning considerations, as collision-free constraints require specific configurations of the robot to cover a position in free space.

\section{Optical Sensor}

The optical sensor which is mounted on top of the robotic platform is used to measure the distance to objects in the environment surrounding the robot. This information is used in a SLAM algorithm to create a map of the environment which is needed for navigation and to determine the possible measurement locations. We considered two different types of sensors for this task: an RGB-D sensor (Microsoft Kinect) and a scanning laser range finder (Hokuyo URG-04LX). The RGB-D sensor provides color (red, green and blue) as well as depth information, which can be used to generate the needed maps in three dimensions. According to its specifications the depth sensor ranges from $1.2 \mathrm{~m}$ to $3.5 \mathrm{~m}$ and its opening angle is $57^{\circ}$. The URG-04LX is a planar scanning laser range finder, that takes 683 samples on a broad opening angle of $240^{\circ}$ at a rate of $10 \mathrm{~Hz}$. Following the specification sheet its guaranteed range is between $0.06 \mathrm{~m}$ and $4 \mathrm{~m}$ and the measurement accuracy varies $\pm 0.01 \mathrm{~m}$ for measurements in the range between $0.02 \mathrm{~m}$ and $1 \mathrm{~m}$, and $\pm 0.001 \mathrm{~m}$ for measurements between $1 \mathrm{~m}$ and $4 \mathrm{~m}$.

Although it just provides two-dimensional data, we decided to use the scanning laser range finder for our experiments, as it is more stable under daylight conditions (e.g. in halls with large windows). Moreover, its output data requires less processing, but still provides sufficient data for our purposes. Due to its wide range, it also enables a more efficient path planning, as no turns need to be explicitly considered to perceive more of the environment's structure.

\section{Ground Truth Tracking}

To provide an accurate ground truth validation for localization, a commercial motion tracking system (Vicon) has been utilized. Consisting of 16 infrared cameras, it yields sub-millimeter accuracy at a rate of $100 \mathrm{~Hz}$, determining the position and orientation of an object equipped with infrared strobes.

\section{E. System Setup and Software Framework}

A system overview of the prototype is shown in Fig. 8. The physical component ("Slider") is comprised of the optical sensor, the magnetic sensor and the robot's locomotion subsystem (wheels and motors). Three threads (localization and mapping, path planner, robot controller) form a feedback loop, whereas the fourth thread (configuration space mapper) records samples and monitors coverage. All threads and their communication are realized within the ROS (robotic operating system) framework.

\section{EXPERIMENTS AND DISCUSSION OF RESULTS}

In a set of initial experiments we have investigated and compared the location accuracy of the raw odometry, which is based on the robot's wheel encoders, and the location accuracy obtained by combining this raw odometry with sensor data from the on-board laser in a SLAM implementation. For this purpose we have equipped the robot with tracking markers and have utilized the optical motion tracking system described in Section III-D to obtain ground truth. Fig. 9 and Fig. 10 show that the raw odometry (dotted blue line) exhibits significant

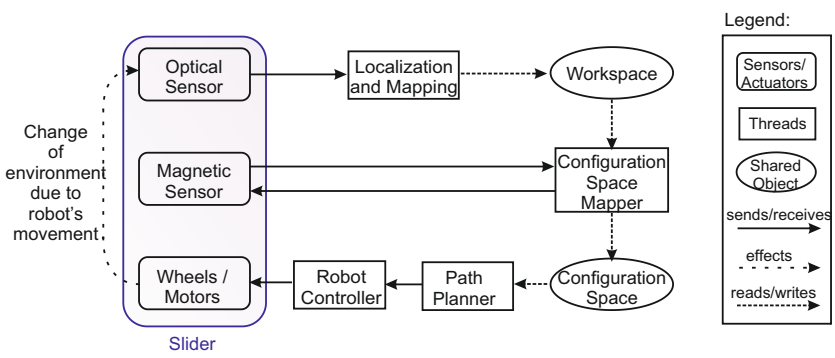

Fig. 8. The system diagram shows the feedback loop formed by the optical sensor on-board the robot (Slider) and the three threads (localization and mapping, path planner, robot controller), with the robot's locomotion subsystem (wheels and motors). The fourth thread (configuration space mapper) records samples and monitors coverage. 


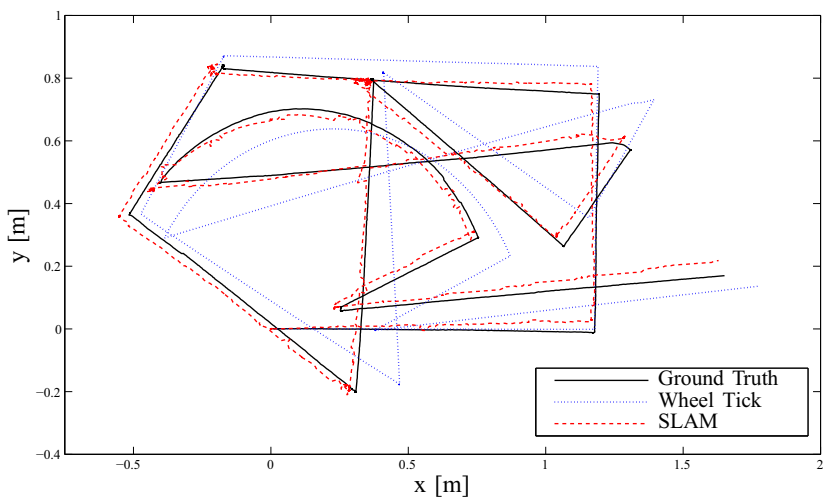

Fig. 9. Trajectories for a robot run. The solid line in black shows the ground truth trajectory. The position estimated by the wheel encoder is illustrated by the dotted blue line. This estimation combined with an optical SLAM algorithm results in the trajectory shown in dashed red.

location error after very short time. In contrast, the SLAMbased position estimate (red line) remains accurate with less than $0.07 \mathrm{~m}$ of error over time.

In order to validate the feasibility of the overall sampling task we have chosen an office environment, due to its challenging nature in terms of fairly fine-grained geometry of obstacles and navigable space.

We utilized a spatial resolution of the sampling grid of $0.05 \mathrm{~m}$ in both horizontal directions. Furthermore, in this experiment we partitioned the robot's configuration space into 12 discrete directions, i.e. $30^{\circ}$ rotational step size. The experimental setup used for the results presented here has been an office space of $4 \mathrm{~m} \times 6 \mathrm{~m}$ and approximately $8 \mathrm{~m}^{2}$ of reachable space. Without any a priori knowledge the robot achieved complete coverage in 63 minutes of driving time. All computations were carried out on-board the robot and during the experiment. Fig. 11 depicts the model of the workspace learned by the robot during the experiment. The estimated trajectory of the robot is shown in Fig. 12. We see that whereas all grid cells have been measured at least once, only few positions in the grid are covered multiple times, resulting in complete coverage with little redundancy, as intended.

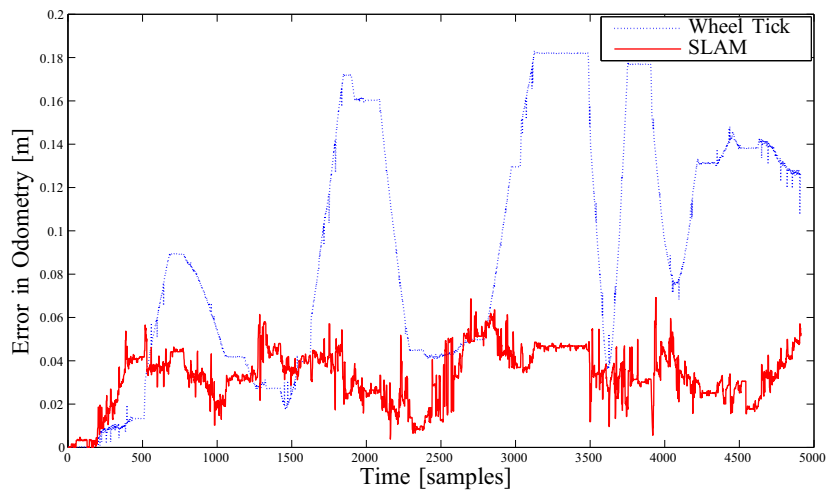

Fig. 10. Error of the estimated trajectory. Dashed blue represents the odometry by the wheel encoder and solid red the optical SLAM.

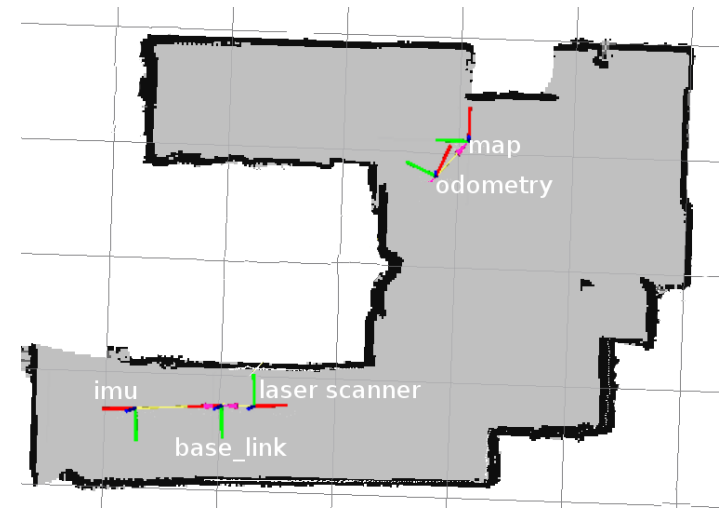

Fig. 11. Final workspace for an experiment within an office at DLR. Black indicates occupancy, gray shows recognized free space. The transformation frames illustrate the estimated position of the robot's center and its sensors, as well as the corrected odometry by the SLAM algorithm (odometry to map).

In Fig. 1 the resulting trajectories and magnetic measurements (shown is the intensity of the magnetic field) are superimposed onto a rectified overhead picture of the office. We see how well the office space is covered. Note that the apparent jumps in the magnetic field result from offsets in the uncalibrated magnetic sensor. We further note that the full pose of the robot is estimated which yields not only the magnetic intensity but results in full observability of the field vector.

Fig. 13 shows further, that $95 \%$ percent coverage was achieved after 50 minutes exploration. The experiment terminated after 63 minutes as $100 \%$ coverage was reached.

\section{CONCLUSIONS AND OUTLOOK}

The presented experimental results show the feasibility of sampling a physical process (here the magnetic field) with high spatial resolution by an autonomous robot. The combination of an optical SLAM algorithm and various graph based path

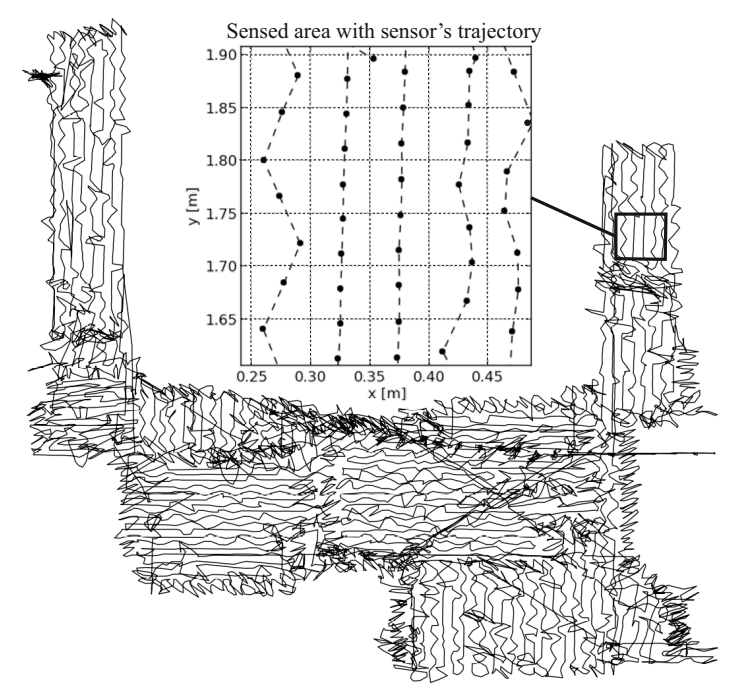

Fig. 12. Estimated trajectory of a sensor capturing the magnetic field within an office at DLR. The zoomed illustration shows that at least one sample was taken in each cell. 


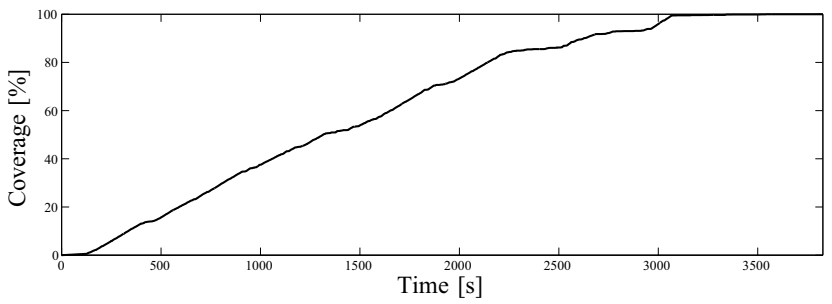

Fig. 13. Illustration of the percentage of covered area over time.

planning algorithms achieve complete coverage and efficient trajectories with little redundancy. In particular a Best-FirstSearch and an adaption to the Dijkstra's algorithm have been applied for an efficient exploration in terms of time and completeness. The robot is capable of autonomously exploring an a priori unknown indoor environment. It successfully perceives and represents the accessible workspace in the form of its configuration space. By planning within this configuration space, collision avoidance can be achieved, as observed occupied space is captured by an optical sensor during exploration and considered implicitly within the robot's configuration space.

We have validated our approach by realizing a fully autonomous ground robot to efficiently produce high resolution magnetic maps of previously uncharted buildings with complete coverage. The quality of the results suggests that this system may be able to economically obtain suitable magnetic maps for pedestrian localization as presented in previous work [2].

Several avenues exist for future improvements of the presented approach. To reduce the time to sample at a given spatial resolution, it is worthwhile to investigate how to compute more efficient paths. Path efficiency may be improved by further preventing redundancy in visited locations as well as improved modeling of the robot's dynamic behavior and associated (time) cost of movements.

Depending on the application domain and intended usage of the obtained data, significant gains may be achieved by more elaborate models of the underlying physical phenomena and adaptive adjustment of spatial sampling frequencies. Such models may include Gaussian Processes [11] and predict values at still unvisited locations to reduce the amount of spatial locations that need to be visited for a complete representation.
Furthermore, multiple refinements of the mechanical platform, such as weight reduction and more agile dynamics, as well as its sensors, such as increased range or mechanical sweeping may significantly improve economic feasibility.

We believe that a cost-efficient and simple to operate platform is very desirable in order to achieve wide-scale adoption for both commercial and scientific purposes that will lead to a much deeper understanding of physical phenomena at previously neglected spatial resolutions.

\section{ACKNOWLEDGMENTS}

The authors would like to thank Lukas Magel for his valuable assistance during the experiments.

\section{REFERENCES}

[1] M. Angermann, M. Doniec, M. Frassl, B. Julian, and P. Robertson, "Characterization of the indoor magnetic field for applications in localization and mapping," in International Conference on Indoor Positioning and Indoor Navigation, Sydney, Australia, Nov 2012.

[2] M. Frassl, M. Angermann, M. Lichtenstern, P. Robertson, B. Julian, and M. Doniec, "Magnetic maps of indoor environments for precise localization of legged and non-legged locomotion," in Intelligent Robots and Systems (IROS), 2013 IEEE/RSJ International Conference on, Nov 2013, pp. 913-920.

[3] H. Cao, J. Chen, L. Dou, H. Fang, Y. Mao, and H. Zhang, "Combined complete coverage path planning for autonomous mobile robot in indoor environment," in ASCC 2009. 7th Asian Control Conference, 2009.

[4] W.H. Huang, "Optimal line-sweep-based decompositions for coverage algorithms," in Robotics and Automation, 2001. Proceedings 2001 ICRA. IEEE International Conference on, 2001.

[5] S. Yang. and C. Luo, "A neural network approach to complete coverage path planning," Systems, Man, and Cybernetics, Part B: Cybernetics, IEEE Transactions on, vol. 34, no. 1, pp. 718-724, Feb 2004.

[6] C. Hofner and G. Schmidt, "Path planning and guidance techniques for an autonomous mobile cleaning robot," in Intelligent Robots and Systems '94. 'Advanced Robotic Systems and the Real World', IROS '94. Proceedings of the IEEE/RSJ/GI International Conference on, Sep 1994.

[7] H. H. Viet, V.-H. Dang, M. N. U. Laskar, and T. Chung, "BA*: an online complete coverage algorithm for cleaning robots," Applied Intelligence, vol. 39, no. 2, pp. 217-235, 2013.

[8] K. Thiayagarajan and C. G. Balaji, "Traversal algorithm for complete coverage," Computer Science, vol. 8, no. 12, pp. 2032-2041, 2012.

[9] G. Grisetti, C. Stachniss, and W. Burgard, "Improving grid-based slam with rao-blackwellized particle filters by adaptive proposals and selective resampling," in ICRA, 2005, pp. 2443-2448.

[10] S. M. LaValle, Planning Algorithms. Cambridge, U.K.: Cambridge University Press, 2006.

[11] A. Viseras Ruiz, M. Angermann, M. Frassl, I. Wieser, and J. Mueller, "Efficient multi-agent exploration with gaussian processes," 2014, submitted. 